\title{
Early Immune Disorders and Neutrophil Activation Induced By Childhood Obesity; CD66b and Myeloperoxidase
}

\author{
Sahar AF. Hammoudah ${ }^{1}$, Azza M. Abdu Allah ${ }^{2 *}$, Mohamed Attia ${ }^{1}$, \\ Eman R El Fiky ${ }^{1}$ \\ ${ }^{1}$ Clinical and Chemical Pathology Department, Faculty of Medicine, Tanta University, Egypt \\ ${ }^{2}$ Medical Biochemistry Department, Faculty of Medicine, Menoufia University, Egypt \\ Corresponding Author: Azza M. Abdu Allah
}

\begin{abstract}
Neutrophils are major source of inflammatory and pro-inflammatory cytokines which play an important role in obesity related cardio-metabolic complications. Little is known about neutrophil activation markers in childhood obesity. Therefore, we investigated the hypothesis that, the component of the innate immune system is activated in childhood obesity through assessment of the expression of CD66b as a specific marker of neutrophil activation and plasma MPO which is a product of activated neutrophil. Ninety children aged 5-15 years (60 obese and 30 age and sex matched healthy controls) were included in the present study. All were subjected to anthropometric measurements, routine laboratory investigations, plasma myeloperoxidase $(M P O)$, complete blood picture $(C B C)$ with examination of peripheral blood smears stained with Giemsa. Surface expression of degranulation marker CD66b on neutrophil surface from peripheral blood was assessed through flow cytometry. We observed that, total WBCs count and total neutrophils count, hs-CRP, and MPO were significantly increased in obese children compared to control group. Although Cd66b expression showed no statistically significant difference, mean fluorescent intensity (MFI) of CD66b positive cells was significantly increased in obese. Our data showed that CD66b, MFI and MPO are major neutrophil activation markers. It also proved that, the innate immune system is activated in childhood obesity.
\end{abstract}

Date of Submission: 04-07-2017

Date of acceptance: $15-07-2017$

\section{Introduction}

Obesity is a growing worldwide health problem (1). The prevalence and severity of childhood obesity has been markedly increased over the past 30 years (2). The prevalence of childhood obesity in the Middle East and North Africa was reported to be high. In Egypt, in children aged 13-15 years, $7 \%$ were reported to be obese and $32.5 \%$ as over-weight (1).

The hypertrophied adipocytes in obese humans were shown to be infiltrated by abundant immune cells like macrophage and lymphocytes which increase the secretion of proinflammtory cytokines resulting in a lowgrade inflammatory condition. Low-grade chronic inflammatory status has been associated with childhood obesity; exactly like adult obesity; which is a key point in the pathogenesis of many metabolic disorders (3-5). Also obesity was reported to be associated with acute inflammation in a sample of adolescents (6).

Neutrophils have strong anti-microbial activity through proteins stored in the specific granules such as myeloperoxidase (MPO) which is secreted after neutrophil activation. MPO generate reactive oxidants and radicals that result in harmful oxidative damage to lipids, lipoproteins and proteins. Also neutrophil activation is associated with mobilization of CD66b from the secondary granules to the outer cell membrane (7).

CD66b (carcinoembryonic antigen-related cell adhesion molecule-8) is expressed only in cells of the granulocytic lineage and is identified as a granulocyte-specific activation antigen (8). Neutrophil activation can be assessed by evaluation of phagocytosis, oxidative burst, and secretion of the pre-formed proteins conserved in secretory granules. Neutrophils from obese children have higher oxygen reactive species, indicating oxidative burst, and greater phagocytosis (9).

We aimed to clarify the hypothesis that, the component of the innate immune system is activated in childhood obesity through assessment of the expression of CD66b as a specific marker of neutrophil activation and plasma MPO which is a product of activated neutrophil. 
Subjects:

\section{Subjects And Methods:}

This case-control study included (90) children (49 males and 41 females), age ranged from 5 to 15 years; they were classified into two groups (60 obese and 30 lean). Subjects were recruited from outpatient clinic of Clinical Pathology department, Tanta University Hospital Egypt, from January 2016 to March 2017. All subjects were subjected to complete history taking and physical examination including anthropometric measurements, plasma myeloperoxidase (MPO), total cholesterol (TC), triglycerides (TG), fasting blood glucose, glycated hemoglobin (HbA1c), hs-CRP, complete blood picture (CBC) with examination of peripheral blood smears stained with Giemsa. Surface expression of degranulation marker CD66b on neutrophil surface from peripheral blood was assessed through flow cytometry.

\section{Sample collection:}

Written informed consent signed by the parents was taken before sample collection (approved by ethical Committee of Human Research, at Tanta University) from all studied subjects. Children with chronic or acute illnesses within the last two weeks or those with hs-CRP more than $9 \mathrm{mg} / \mathrm{l}$ were excluded from the study.

\section{Detailed medical history and full clinical assessment:}

Anthropometric and body composition measurements: will be performed in all study participants before breakfast, with the subject wearing light clothing without shoes. For all subjects, weight and height were measured to the nearest $0.1 \mathrm{~kg}$ and $0.1 \mathrm{~cm}$, respectively, and the BMI was calculated. BMI is plotted on a BMI chart in which children is standardized by sex and age. Plotting the BMI yields a percentile, and this is used to classify a child's weight status according to the reference tables of the Centers for Disease Control and Prevention (CDC) into: or normal weight BMI (10th - 85th percentiles), obese (equal or more than the 95th percentile) (10) .

\section{Sampling and biochemical parameters assay procedure:}

Fasting peripheral blood samples will be taken under complete aseptic conditions with sterile disposable syringes, where $4 \mathrm{ml}$ will be collected in EDTA vacutainer tube for CBC, Geimsa stained smears, plasma separation and flow cytometric analysis. $2 \mathrm{ml}$ collected into an empty sterile tube and allowed to be clotted and serum was separated for measurement of hs-CRP, serum fasting glucose, cholesterol and triglycerides levels Total leukocyte counts were obtained using an automated cell counter (ERMA PCE-210N). Blood smears were examined for differential leukocyte. HsCRP was assessed by immuno-turbidimetry (QCA S.A. Amposta, Spain, $0.02 \mathrm{mg} / \mathrm{L}$ sensitivity). Serum triglyceride, total cholesterol, and glucose levels were determined using an enzymatic-colorimetric test (QCA S.A., Amposta, Spain). HbA1c was calculated by application of the equation $\left(\mathrm{eAG}(\mathrm{mg} / \mathrm{dl})=\left(28.7^{*} \mathrm{HbAlc}\right)-46.7\right)(11)$ Plasma concentrations of MPO was assessed using sandwich enzyme-linked immunosorbent assays (HyCult biotechnology, Uden, the Netherlands). All plasma samples were analyzed in the same run. The intra- and interassay coefficients of variance of the various assays were $<10 \%$.

\section{Flow Cytometry analysis:}

Neutrophils inflammatory status in all included children was assessed by measuring surface expression of degranulation marker CD66b through flow cytometry. Whole blood (100 $\mu \mathrm{l})$ (EDTA) was incubated with a fluorescein isothiocyanate-labeled antibody against CD66b. Phosphate buffered saline and a fluorescein isothiocyanate-labeled isotype antibody were used as negative control. After $30 \mathrm{~min}$ of incubation in the dark, erythrocytes were lysed and the vials were centrifuged at $400 \mathrm{~g}$. The supernatant was discarded and the cells were washed twice with phosphate buffered saline- $0.1 \%$ bovine serum albumin. Finally, cells were resuspended in $1 \%$ paraformaldehyde solution and fluorescence activated cell sorting analysis was performed the same day on the FACSCalibur using Cellquest software (Becton Dickinson, Franklin lakes, NJ). Data are expressed as mean fluorescence intensity.

\section{Statistical analysis}

Statistical analyses were conducted using IBM SPSS software package version 20.0. (Armonk, NY: IBM Corp).The Kolmogorov- Smirnov, Shapiro and D'agstino tests were used to verify the normality of distribution of variables. Comparisons between groups for categorical variables were assessed using Chi-square test. Student t-test was used to compare two groups for normally distributed quantitative variables while ANOVA was used for comparing the studied groups and followed Kruskal Wallis test was used to compare different groups for abnormally distributed quantitative variables. Mann Whitney test was used to compare between two groups for abnormally distributed quantitative variables. 


\section{Results}

This study was conducted on 90 children aged $10 \pm 0.7$ (range 5-15), 45.6\% female subjects. Study population was divided into two groups, 60 obese and 30 lean as reference control group. There was a statistically significant difference between the studied groups regarding BMI, serum FBG, HbA1c, TC, TG and hsCRP $(P<0.001)$, while there was no significant difference regarding age and gender. Total WBCs count and total neutrophils count were significantly increased in obese children compared to control group.

\section{Plasma markers of neutrophil activation}

The plasma levels of MPO; as neutrophil activation marker; were significantly increased in obese subjects as compared to healthy control groups, the levels were $29.9 \pm 5.0 \mathrm{ng} / \mathrm{ml}$ and $14.7 \pm 3.3 \mathrm{ng} / \mathrm{ml}$ respectively $(P<0.001)$ (table1). Circulating MPO level showed a significant correlation with neutrophil count $(r=0.551, P<0.001)$, indicating neutrophil activation (table 3 , figure 5$)$.

\section{CD66b expression on neutrophils Interpretation of the results of CD66b:}

The cells of interest (neutrophils) were selected by gating according to forward and side scatter characteristics in the formed dot blot. The percentage of the neutrophils positive for CD66b was determined in relation to the isotype negative control that was run in parallel to the test sample. Mean fluorescent intensity (MFI) was calculated as the mean fluorescence of CD66b of positive cells divided by that of isotype control cells in the histogram plot. Surface CD66b expression was measured in all studies subjects. CD66b neutrophils degranulation marker was expressed by most neutrophils (Table 1). CD66b\% expression in obese was 97.8 (80.2-99.8) and was 97.1 (88.3-99.3) in normal controls. There was no significant statistical difference between studied groups $(\mathrm{P}=0.097)$. In contrast the mean fluorescence intensity of CD66b positive cells was significantly increased in obese [26 (10.9-87.5)] compared to [12 (3.9-43.0)] in control group. (Table 1). No statistical significant correlations between MFI and all studied parameters in obese group. In Control group MFI showed positive correlation only with CD66b expression $(\mathrm{r}=0.456, \mathrm{P}=0.011)$ (table 2)

Table (1): Clinical and laboratory data of studied groups

\begin{tabular}{|c|c|c|c|}
\hline & $\begin{array}{l}\text { Control } \\
(\mathrm{n}=\mathbf{3 0})\end{array}$ & $\begin{array}{c}\text { Obese } \\
(n=60)\end{array}$ & $\mathbf{p}$ \\
\hline Age (years) & $10.1 \pm 3.1$ & $10.6 \pm 3$ & 0.417 \\
\hline \multicolumn{4}{|l|}{ Gender } \\
\hline Male & $13(43.3 \%)$ & $36(60 \%)$ & \multirow{2}{*}{0.134} \\
\hline Female & $17(56.7 \%)$ & $24(40 \%)$ & \\
\hline BMI $\left(\mathrm{kg} / \mathrm{m}^{2}\right)$ & $17.7 \pm 2.5$ & $26.3 \pm 4.7$ & $<0.001$ \\
\hline Fast glucose(mg/dl) & $69.6 \pm 9.9$ & $82.2 \pm 14.4$ & $\langle 0.001$ \\
\hline Total cholesterol (mg/dl) & $75(54-142)$ & $136.5(61-205)$ & $\overline{<0.001}$ \\
\hline Triglycerides (mg/dl) & $62.2 \pm 11.3$ & $89.3 \pm 26.7$ & $\overline{<0.001}$ \\
\hline WBCs count $\left(\times 10^{3} / \mathrm{Cmm}\right)$ & $6.7 \pm 1.7$ & $7.8 \pm 2.1$ & $0.019^{*}$ \\
\hline Neutroph $\left(\times 10^{3} / \mathrm{Cmm}\right)$ & $3.2(1.9-6.2)$ & $4.2(1.6-8.2)$ & $0.008^{*}$ \\
\hline HS-CRP (mg/L) & $1.7(0.7-5.2)$ & $3.6(0.6-8.3)$ & $<0.001^{\prime}$ \\
\hline CD66b (\%) & $97.1(88.3-99.3)$ & $97.8(80.2-99.8)$ & 0.097 \\
\hline MFI Ratio & $12(3.9-43.0)$ & $26(10.9-87.5)$ & $<0.001$ \\
\hline MPO (ng/ml) & $14.7 \pm 3.3$ & $29.9 \pm 5$ & $<0.001^{\prime}$ \\
\hline
\end{tabular}

Qualitative data were described using number and percent and was compared using Chi square test. Normally quantitative data was expressed in mean \pm SD and was compared using student t-test, abnormally distributed data was expressed in median (Min. - Max.) and was compared using Mann Whitney test *: Statistically significant at $\mathrm{p} \leq 0.05$

Table (2): Correlation between different parameters in control group $(\mathrm{n}=30)$

\begin{tabular}{|c|c|c|c|c|c|c|c|c|}
\hline & \multicolumn{2}{|c|}{$\begin{array}{l}\text { Neutroph }(\mathbf{x 1 0 3} \\
\left.\times 10^{3} / \mathrm{Cmm}\right)\end{array}$} & \multicolumn{2}{|c|}{ HS-CRP (mg/L) } & \multicolumn{2}{|c|}{ CD66b (\%) } & \multicolumn{2}{|c|}{ MFI Ratio } \\
\hline & $\mathbf{r}_{\mathrm{s}}$ & p & $\mathbf{r}_{\mathrm{s}}$ & $\mathbf{P}$ & $\mathbf{r}_{\mathrm{s}}$ & p & $\mathbf{r}_{\mathrm{s}}$ & p \\
\hline Neutroph $\left(\mathrm{x10}^{3} / \mathrm{Cmm}\right)$ & 1.000 & & 0.467 & 0.009 & 0.023 & 0.902 & -0.014 & 0.939 \\
\hline HS-CRP (mg/L) & $0.467^{*}$ & $0.009^{*}$ & 1.000 & . & -0.320 & 0.085 & -0.289 & 0.122 \\
\hline CD66b $(\%)$ & 0.023 & 0.902 & -0.320 & 0.085 & 1.000 & 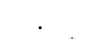 & $0.456^{*}$ & $0.011^{*}$ \\
\hline MFI Ratio & -0.014 & 0.939 & -0.289 & 0.122 & $0.456^{*}$ & $0.011^{*}$ & 1.000 & . \\
\hline BMI $\left(\mathrm{kg} / \mathbf{m}^{2}\right)$ & 0.076 & 0.688 & 0.016 & 0.934 & -0.124 & 0.512 & -0.051 & 0.790 \\
\hline MPO (ng/ml) & 0.269 & 0.151 & -0.083 & 0.663 & -0.120 & 0.528 & -0.028 & 0.882 \\
\hline
\end{tabular}

$\mathrm{r}_{\mathrm{s}}$ : Spearman coefficient

*: Statistically significant at $\mathrm{p} \leq 0.05$ 
Table (3): Correlation between different parameters in obese group $(n=60)$

\begin{tabular}{|c|c|c|c|c|c|c|c|c|}
\hline & \multicolumn{2}{|c|}{ Neutroph $\left(\mathrm{x10}^{3} / \mathrm{Cmm}\right)$} & \multicolumn{2}{|c|}{ HS-CRP (mg/L) } & \multicolumn{2}{|c|}{ CD66b (\%) } & \multicolumn{2}{|c|}{ MFI Ratio } \\
\hline & $\mathbf{r}_{\mathrm{s}}$ & $\mathbf{p}$ & $\mathbf{r}_{\mathrm{s}}$ & $\mathbf{p}$ & $\mathbf{r}_{\mathrm{s}}$ & $\mathbf{p}$ & $\mathbf{r}_{\mathrm{s}}$ & $\mathbf{p}$ \\
\hline Neutroph $\left(\mathrm{x10}^{3} / \mathrm{Cmm}\right)$ & 1.000 & - & $0.342^{*}$ & $0.007^{*}$ & 0.221 & 0.090 & 0.152 & 0.247 \\
\hline HS-CRP (mg/L) & $0.342^{*}$ & $0.007^{*}$ & 1.000 & - & 0.037 & 0.778 & 0.193 & 0.140 \\
\hline CD66b (\%) & 0.221 & 0.090 & 0.037 & 0.778 & 1.000 & - & -0.021 & 0.871 \\
\hline MFI Ratio & 0.152 & 0.247 & 0.193 & 0.140 & -0.021 & 0.871 & 1.000 & - \\
\hline BMI $\left(\mathrm{kg} / \mathrm{m}^{2}\right)$ & 0.105 & 0.425 & -0.029 & 0.828 & -0.053 & 0.689 & -0.050 & 0.704 \\
\hline MPO (ng/ml) & $0.551^{*}$ & $<0.001^{*}$ & 0.226 & 0.082 & -0.021 & 0.874 & 0.169 & 0.704 \\
\hline
\end{tabular}

$\mathrm{r}_{\mathrm{s}}$ : Spearman coefficient

*: Statistically significant at $\mathrm{p} \leq 0.05$

Figure (1) Show the Flow Cytometric analysis of a participant

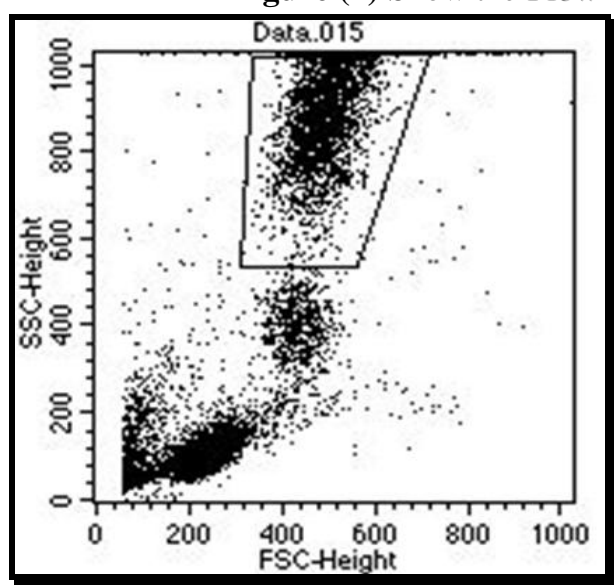

Figure (1a): Dot plot showing Gated granulocytes

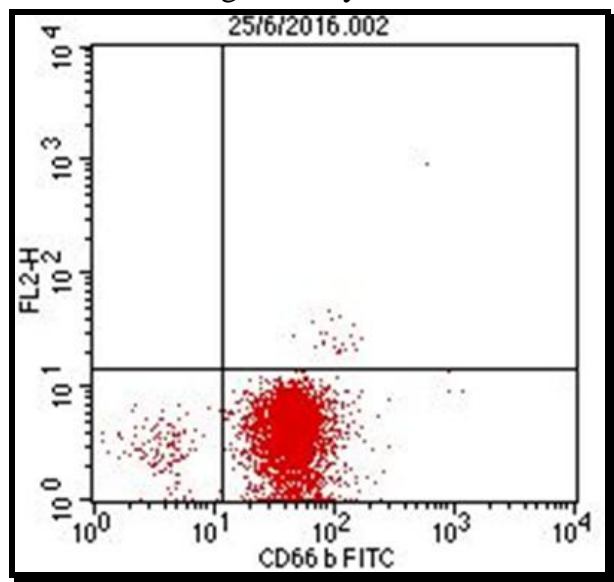

Figure (1c) Dot plot showing CD66b positive

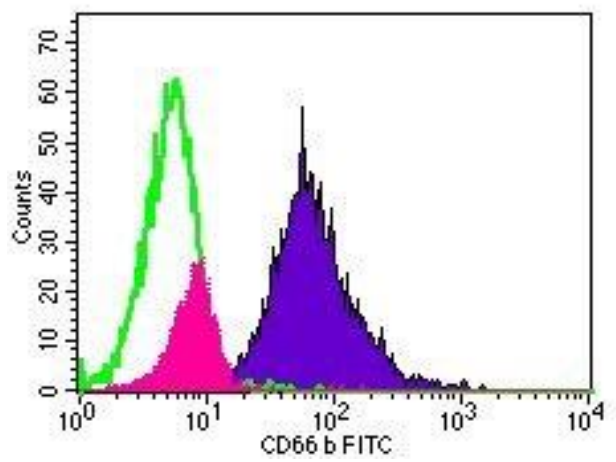

Figure (1e) showing MFI of CD66b of one of the obese patients

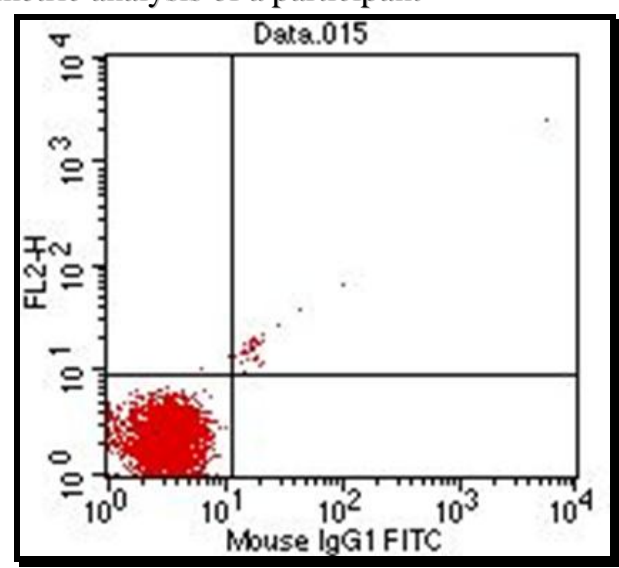

Figure (1b): Dot plot showing Negative control

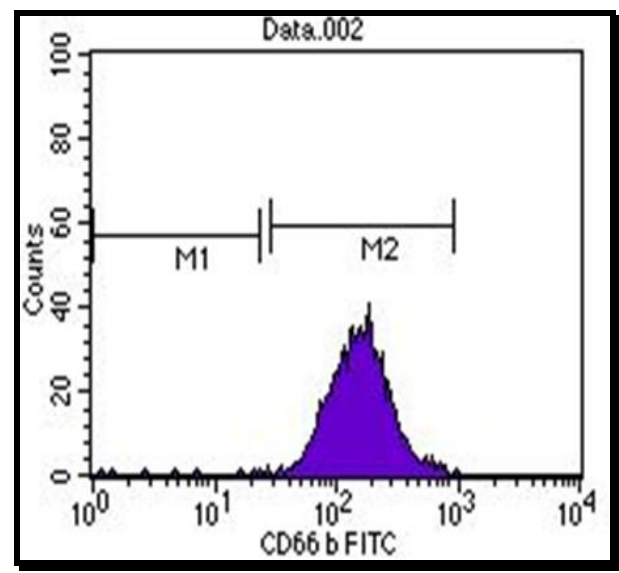

Figure (1d): Dot plot showing CD66b MFI ratio 


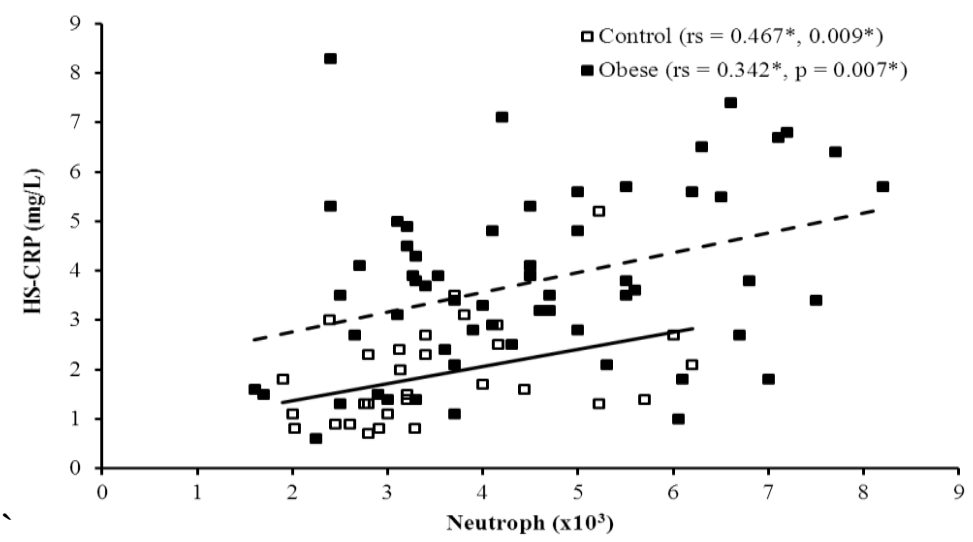

Figure (2): Correlation between Neutrophil count and HS-CRP in each group

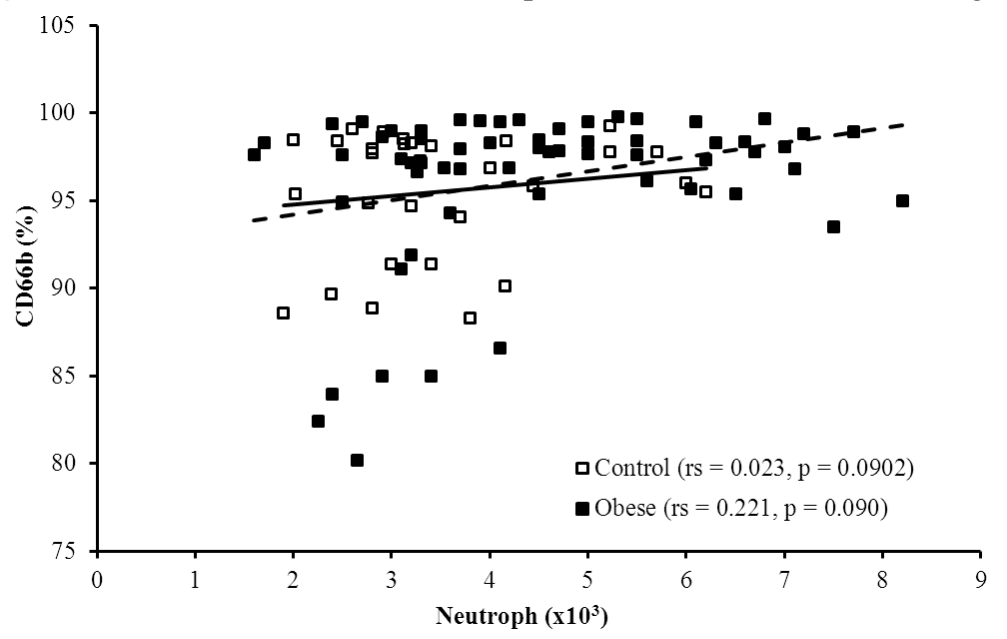

Figure (3): Correlation between Neutrophil count and CD66b (\%) in each group

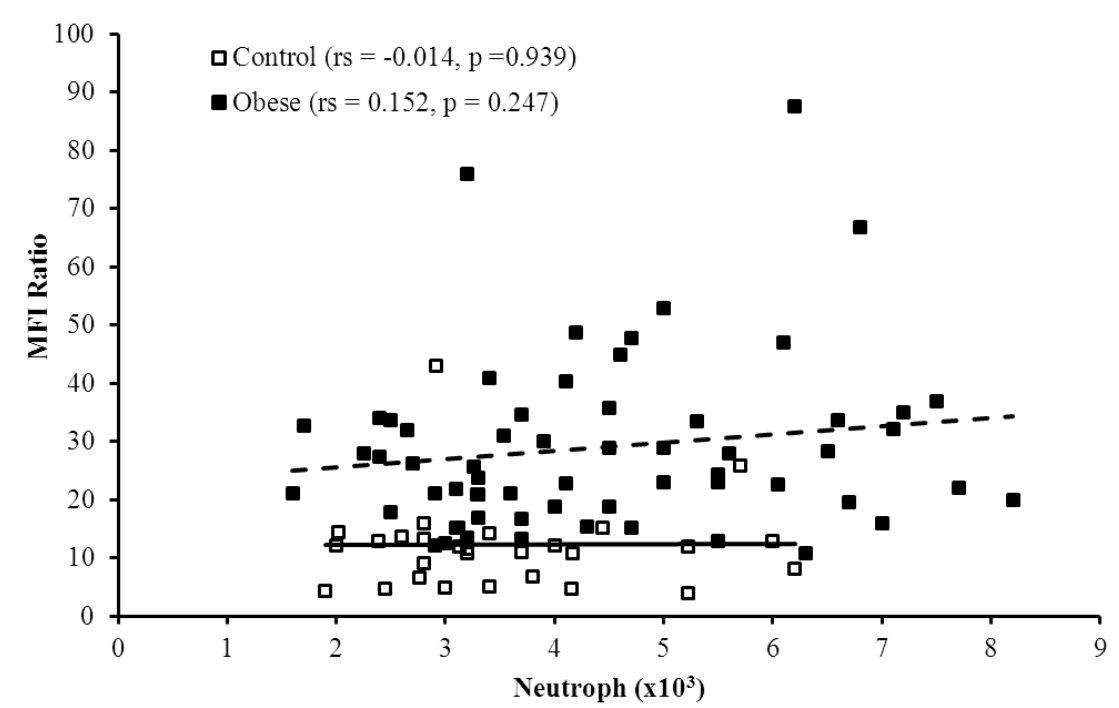

Figure (4): Correlation between Neutrophil count and MFI ratio in each group 


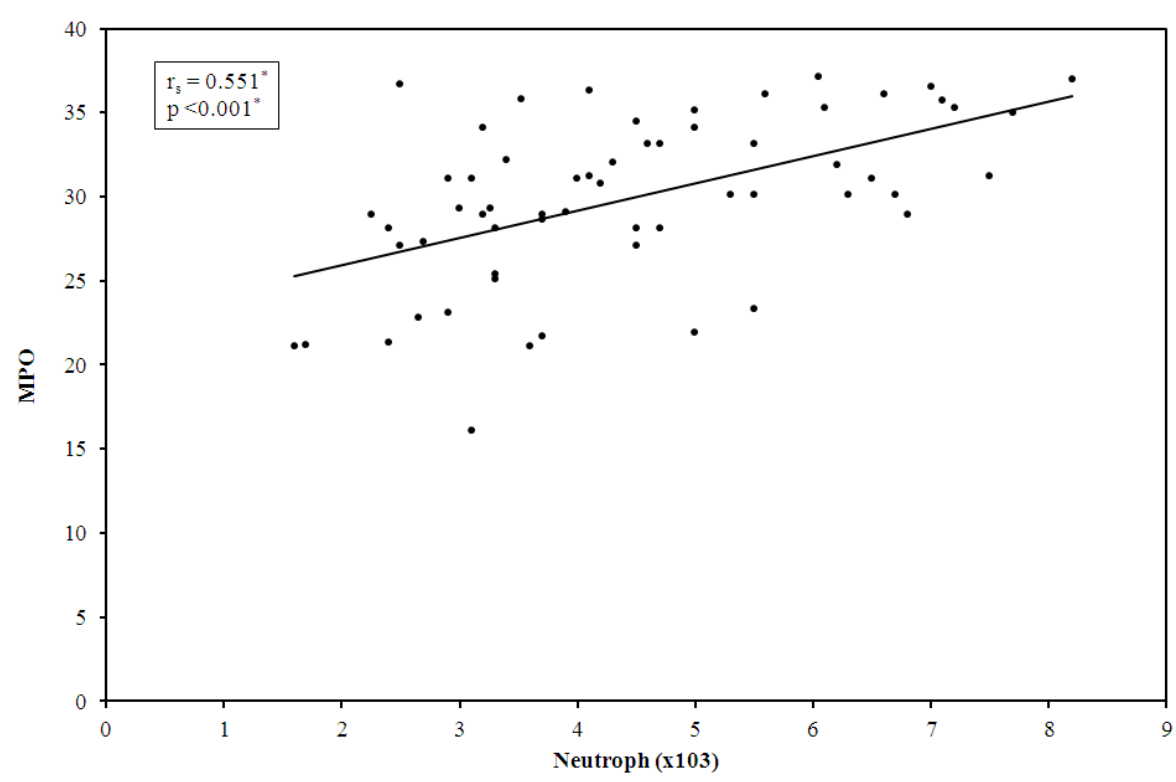

Figure (4): Correlation between Neutrophil count and MPO in obese group

\section{Discussion:}

Childhood is an important time for immune system development. Childhood obesity is associated with increase risk of immune-mediated morbidities like Diabetes mellitus, asthma, non-alcoholic fatty liver and cardiovascular diseases. Over expression of pro-inflammatory cytokines; like TNF $\alpha$ and Interferon $\gamma$; and activated innate immune cells play a central in the development complications (12). It has been reported that, human adipose tissue from lean subjects secretes anti-inflammatory cytokines while obese individual adipose tissue expresses pro-inflammatory cytokines such as interleukin-6 and TNF-a, potentially inducing low-grade systemic inflammation $(7,13,14)$. Role and mechanisms of neutrophil activation in childhood obesity needed to be evaluated since neutrophils are playing a central role in innate immunity. So we aimed to determine the degree of neutrophil activation by measuring plasma levels of MPO and neutrophil membrane expression of CD66b.

In the present study we observed no significant statistical difference between the obese children and control group regarding age and gender. These data were in accordance with data reported by Palhares HM et al., 2017 (15). As regard serum FBG, HbAlc, TC, TG, hsCRP and BMI there was a statistical significant difference between the two studied groups. Similar data were reported by Kelishadi et al., 2012 (16), Hanh et al., 2017 (17).These data indicated that there must be a social awareness about childhood obesity as an important risk factor for cardio-metabolic complications. The results of the present study showed that total WBCs count and total neutrophils count were significantly increased in obese children compared to control group. Similar results in children were reported by Visser et al., 2011 (18), Zaldivar et al., 2006 (19) and in young adults by Yoshimura et al., 2015 (5).

These data supported the theory that obesity is a low grade inflammatory status since neutrophils are known to be important effectors in inflammation. Neutrophils react to inflammation by degranulation of their cytoplasmic granules. Activated neutrophils are capable of producing many cytokines and chemokines that promote activation and recruitment of macrophages, dendretic cells and lymphocytes $(20,21)$. In our current study, the plasma levels of MPO were significantly increased in obese subjects as compared to healthy control groups. Nijhuis et al., 2009 (7) reported similar data in adults with morbid obesity. These results supported the fact that, MPO is a pleiotropic inflammatory cytokine produced by polymorph nuclear neutrophils and macrophages and secreted during neutrophils activation $(7,22)$. Olza et al., $2012(23)$ in a prospective multicentre case control study on 446 children aged 6-12 years showed that MPO was statistically significant increased in obese children compared to normal. They stated that, MPO was associated with markers of endothelial dysfunction which indicate that MPO is an early biomarker of inflammation associated with CVD risk in obese children. Total WBCs count and total neutrophils count were significantly increased in obese children compared to control group. These results were in accordance with Zaldivar et al., 2006 (19). Yoshimura et al., 2015 (5) reported similar data in young adults. These data supported the role of neutophils in inflammatory status in obesity. Since the life span of neutrophil is short, continuous activation of innate immunity is suggested and consequently low grade chronic inflammatory status is associated with obesity. In 
the present study CD66b; neutrophils degranulation marker; was expressed by most neutrophils. There was no significant statistical difference between obese and control groups. In contrast the mean fluorescence intensity of CD66b positive cells was significantly increased in obese children compared to control group. (Figure 3 ). Our results were in accordance with Reyes et al., 2015 (6) who studied adulthood obesity in 528 adolescents (16.8 y old, 47\% females). Nijhuis et al., 2009 (7) reported that Cd66b membrane expression was significantly increased in obsess compared control $\mathrm{P}<0.01$. This contrast to our result might be explained that they study morbid obese adult subjects, which is quite different population from our cases $(6,7)$.

The involvement of neutrophils in chronic inflammatory status in obesity attracted more attention especially in children. Genome-wide gene expression analysis on obese young adults showed significant expression of two genes (neutrophil elastase and MPO) which are considered as very important biomarkers of neutrophil activation (24). Increased neutrophil counts, high levels of soluble MPO and elevated MFI of CD66b in obese children compared to control proved that, the innate immune system is activated in childhood obesity. It also clarifies the essential role of neutrophil activation in the pathogenesis of obesity and its related co-morbidities. Our data showed that CD66b MFI and MPO are major neutrophil activation markers.

\section{Conclusion}

Further studies on CD66b might contribute to the development of novel therapeutic modalities that reduce the severity of obesity-related co-morbidities.

\section{References}

[1] WHO (2014): Global school-based student health survey. Online database.Geneva:WHO, 2014. (Accessed 25 January 2014, at http://www. who.int/chp/gshs/en/.)

[2] de Onis M, Blossner M, Borghi E. Global prevalence and trends of overweight and obesity among preschool children. Am J Clin Nutr. 2010; 92: 1257-64.

[3] Gregor M. F. and Hotamisligil G. S. "Inflammatory mechanisms in obesity," Annual Review of Immunology. 2011; 29: 415-445.

[4] Hotamisligil G. S. "Inflammation and metabolic disorders," Nature. 2006; 444 (7121): 860-867.

[5] Yushimora A, Ohnishi S, Orito C, Kawahara Y, Takasaki H, Takeda H, Sakamoto N,Hashino S. Association of Peripheral Total and Differential Leukocyte Counts with Obesity-Related Complications in Young Adults. Obes Facts 2015; 8:1-16.

[6] Reyes M, Quintanilla C, Burrows R, Blanco E, Mariana Cifuentes M, and Gahagan S. Pediatr Diabetes. 2015 March ; 16(2): 109116.

[7] Nijhuis J, Rensen SS, Slaats Y, van-Dielen FMH, Buurman WA and Grever JWM. Neutrophil Activation in Morbid Obesity, Chronic Activation of Acute Inflammation. Obesity. 2009; 17: 2014-2018.

[8] Thompson JA, Grunert F, and Zimmermann W. Carcinoembryonic antigen gene family: molecular biology and clinical perspectives. J. Clin. Lab. Anal.1991; 5: 344-366.

[9] Nieman DC, Henson DA, Nehlsen-Cannarella SL et al. Influence of obesity on immune function. J Am Diet Assoc. 1999; 99: 294299.

[10] Barlow SE . Expert committee recommendations regarding the prevention, assessment, and treatment of child and adolescent overweight and obesity: summary report. Pediatrics. 2007;120 (14): S164-S192.

[11] David M. Nathan ,Judith Kuenen, Rikke Borg,Hui Zheng, David Schoenfeld, and Robert J. Heine. Translating the A1C Assay Into Estimated Average Glucose Values .Diabetes Care 2008 Aug; 31(8): 1473-1478

[12] Mattos RT, Medeiros NI, Menezes CA, Fares RCG, Franco EP, Dutra WO, FR Santos, Oliveira RC, Gomes JAC. Chronic LowGrade Inflammation in Childhood Obesity Is Associated with Decreased IL-10 Expression by Monocyte Subsets. PLoS One. 2016; 11(12).

[13] Utsal L, Tillmann V, Zilmer M, Maestu J, Purge P, Jurimae J, et al. Elevated serum IL-6, IL-8, MCP-1, CRP, and IFN-gamma levels in 10- to 11-year-old boys with increased BMI. Horm Res Paediatr. 2012;78(1):31-9.

[14] Ogden CL, Carroll MD, Kit BK, Flegal KM. Prevalence of childhood and adult obesity in the United States, 2011-2012. JAMA. 2014; 311:806-14.

[15] Heloisa Marcelina da Cunha Palhares, Adriana Paula da Silva, Daniela Cristina Silva Resende, Gilberto deAraujo Pereira, Virmondes Rodrigues-Junior, Maria de Fatima Borges. Evaluation of clinical and laboratory markers of cardiometabolic risk in overweight and obese children and adolescents. CLINICS 2017;72(1):36-43

[16] Kelishadi R, Hashemipour M, Sheikh-Heidar A, Ghatreh-Samani S: Changes in serum lipid profile of obese or overweight children and adolescents following a lifestyle modification course. ARYA Atherosclerosis Journal. 2012, 8(3): 143-148

[17] Hanh NTH, Tyet LT, Doa DTA,Tao Y and Chu DT. Childhood Obesity Is a High-risk Factor for Hypertriglyceridemia: A Casecontrol Study in Vietnam. Osong Public Health Res Perspect. 2017 Apr; 8(2): 138-146.

[18] Visser M, Bouter LM, McQuillan GM, Wener MH, Harris TB: Low-grade systemic inflammation in overweight children. Pediatrics. 2001; 107:E13.

[19] Zaldivar F, McMurray RG, Nemet D, Galassetti P, Mills PJ, Cooper DM: Body fat and circulating leukocytes in children. Int J Obes (Lond). 2006; 30: 906-911.

[20] Mantovani A, Cassatella MA, Costantini C and Jaillon S .Neutrophils in the activation and regulation of innate and adaptive immunity. Nature Reviews. Immunology 2011; 519-531.

[21] Amulic B, Cazalet C, Hayes GL, Metzler KD \& Zychlinsky A 2012 Neutrophil function: from mechanisms to disease. Annual Review of Immunology.2012;30:459-489.

[22] Nussbaum C, Klinke A, Adam M, Baldus S, Sperandio M. Myeloperoxidase: a leukocyte-derived protagonist of inflammation and cardiovascular disease. Antioxid Redox Signal. 2013 ; 18(6):692-713.

[23] Olza J, Aguilera CM, Gil-Campos M, Leis R, Bueno G, Martínez-Jiménez MD, Valle M, Cañete R, Tojo R, Moreno LA, Gil A. Myeloperoxidase is an early biomarker of inflammation and cardiovascular risk in prepubertal obese children. Diabetes Care. 2012;35(11):2373-6.

[24] X Xu, S Su, X Wang, V Barnes, C De Miguel, D Ownby, J Pollock, H Snieder, W Chen and X Wang. Obesity is associated with more activated neutrophils in African American male youth. International Journal of Obesity.2014;19. 